Angiogenic response of locally advanced breast cancer to neoadjuvant chemotherapy evaluated with parametric histogram from dynamic contrast-enhanced MRI

This article has been downloaded from IOPscience. Please scroll down to see the full text article. 2004 Phys. Med. Biol. 493593

(http://iopscience.iop.org/0031-9155/49/16/007)

The Table of Contents and more related content is available

Download details:

IP Address: 140.112.113.225

The article was downloaded on 04/03/2009 at 04:05

Please note that terms and conditions apply. 


\title{
Angiogenic response of locally advanced breast cancer to neoadjuvant chemotherapy evaluated with parametric histogram from dynamic contrast-enhanced MRI
}

\author{
Yeun-Chung Chang ${ }^{1,2}$, Chiun-Sheng Huang ${ }^{3}$, Yi-Jui Liu', \\ Jyh-Horng Chen ${ }^{1}$, Yen-Shen $\mathrm{Lu}^{4}$ and Wen-Yih I Tseng ${ }^{2}$ \\ ${ }^{1}$ Department of Electric Engineering, National Taiwan University, Taipei, Taiwan \\ ${ }^{2}$ Department of Medical Imaging, National Taiwan University Hospital and National Taiwan \\ University College of Medicine, Taipei, Taiwan \\ ${ }^{3}$ Department of Surgery, National Taiwan University Hospital and National Taiwan University \\ College of Medicine, Taipei, Taiwan \\ ${ }^{4}$ Department of Oncology, National Taiwan University Hospital and National Taiwan University \\ College of Medicine, Taipei, Taiwan \\ ${ }^{5}$ Department of Automatic Control Engineering, Feng Chia University, Taichung, Taiwan \\ E-mail: wytseng@ha.mc.ntu.edu.tw
}

Received 15 March 2004

Published 2 August 2004

Online at stacks.iop.org/PMB/49/3593

doi:10.1088/0031-9155/49/16/007

\begin{abstract}
The aim of this study was to evaluate angiogenic compositions and tumour response in the course of neoadjuvant chemotherapy in patients with locally advanced breast cancer (LABC) using dynamic contrast-enhanced (DCE) MRI. Thirteen patients with LABC underwent serial DCE MRI during the course of chemotherapy. DCE MRI was quantified using a two-compartment model on a pixel-by-pixel basis. Analysis of parametric histograms of amplitude, exchange rate $k_{\text {out }}$ and peak enhancement over the whole tumour was performed. The distribution patterns of histograms were correlated with the tumour response. Initial kurtosis and standard deviation of amplitude before chemotherapy correlated with tumour response, $r=0.63$ and $r=0.61$, respectively. Comparing the initial values with the values after the first course of chemotherapy, tumour response was associated with a decrease in standard deviation of amplitude $(r=0.79)$, and an increase in kurtosis and a decrease in standard deviation of $k_{\text {out }}$ ( $r=0.57$ and 0.57 , respectively). Comparing the initial values with the values after completing the chemotherapy, tumours with better response were associated with an increase in kurtosis $(r=0.62)$, a decrease in mean $(r=0.84)$ and standard deviation $(r=0.77)$ of amplitude, and a decrease in mean of peak enhancement $(r=0.71)$. Our results suggested that tumours with better response tended to alter their internal compositions
\end{abstract}


from heterogeneous to homogeneous distributions and a decrease in peak enhancement after chemotherapy. Serial analyses of parametric histograms of DCE MRI-derived angiogenic parameters are potentially useful to monitor the response of angiogenic compositions of a tumour throughout the course of chemotherapy, and might predict tumour response early in the course.

\section{Introduction}

Breast cancer is a leading cause of mortality in women (Tabar et al 2003, Chie et al 2002). Patients with locally advanced breast cancer (LABC) have poor prognosis and require multimodality therapy to increase the survival (Heys et al 1994). To date, neoadjuvant chemotherapy is a standard treatment for LABC because of more effective control of local disease and better long-term survival (Weidner et al 1991, Koning and Hart 1998). However, the rate of local control varies significantly between series, depending on factors such as the choice of chemotherapeutic agents, number of cycles in a treatment course, response to treatment selection and clinical status of patients. To tailor the choice of chemotherapy for individuals, objective assessment is desirable to monitor the local tumour response in the course of treatment.

Conventional evaluation of tumour response relies on physical examination and mammography (Pierce et al 1996, Vinnicombe et al 1996). Physical examination depends heavily on examiners' experience and is prone to subjectivity. Mammography is limited by uncertain significance of residual microcalcifications and residual masses on post-induction chemotherapy (Pierce et al 1996). Recently, magnetic resonance imaging (MRI) has shown to be more accurate than other imaging techniques in determining the size and extent of most breast tumours, whether the assessment is made prior to or following therapeutic intervention (Mumtaz et al 1997, Rodenko et al 1996, Boetes et al 1995, Gilles et al 1994, Weatherall et al 2001). Particularly, dynamic contrast-enhanced (DCE) MRI is found useful in differentiating benign from malignant tumours (Kuhl et al 1999) and in detecting residual tumours after chemotherapy (Gilles et al 1994).

DCE MRI is a method of imaging the physiology of microcirculation based on the measurement of enhancement modulation before and after the injection of a contrast agent such as gadolinium chelate. Evidence is mounting that DCE MRI-based measurements correlate well with tumour angiogenesis (Tofts et al 1995, Knopp et al 1999, Taylor et al 1999). These measurements are sensitive to physiology and to the pharmacokinetics of the contrast agent in individual tumours. Moreover, they can present anatomical images of tumour microcirculation at excellent spatial resolution. Sequential assessment of tumour mass and tumour angiogenesis during neoadjuvant chemotherapy provides an opportunity to understand molecular mechanism associated with pathologic response and to study the possibility of guiding appropriate choices for induction treatment.

Most analysis of DCE MRI is restricted to assessing the region of interest on a single slice (Mussurakis et al 1997, Fischer et al 1999, Buadu et al 1996). This approach does not address the heterogeneity of angiogenic compositions within the tumours and is subjected to sampling bias. In this study, a new approach was proposed to assess angiogenic compositions of the whole tumour with the use of DCE MRI-derived parametric histograms. The purposes of our study were: (1) to study whether initial histograms of tumour angiogenic compositions can predict tumour response, (2) to determine the relationships between the tumour response 
and the change in histograms after the whole course of chemotherapy and (3) to study whether changes in histograms early in the course of chemotherapy can predict tumour response.

\section{Materials and methods}

\subsection{Patient recruitment}

Thirteen patients (50.92 \pm 7.95 years of age) of LABC who fulfilled the criterion of tumour staging as $\mathrm{T} 3$ or $\mathrm{T} 4$ according to the American Joint Committee on Cancer (AJCC) were enrolled in our study. None of these patients had distant metastasis before initiating neoadjuvant chemotherapy. Written informed consent was obtained from all patients by the Institutional Review Board of the Hospital. All patients received at least three serial breast MR studies at the following time points: before receiving incision biopsy and initiating chemotherapy, following the completion of the first course of chemotherapy and before final surgical tumour resection $(n=8)$ or core biopsy $(n=5)$. All the 13 cases received incision biopsy after completing the first MR study of breasts. Twelve cases were invasive ductal carcinoma and one case was invasive lobular carcinoma. The chemotherapy regimens included CEF (cyclophosphamide, epirubicin, fluorouracil) in three patients; palcitaxel plus cisplatin in three patients; weekly docetaxel plus oral UFUR (tegafur/uracil) in six patients, in which three of them received AC (doxorubicin, cyclophophamide) later due to unsatisfied response of docetaxel treatment; and vinorelbin plus weekly high dose 5-flurouracil/leucovorin $24 \mathrm{~h}$ infusion in one patient. Patients were followed for $15 \pm 6.9$ months (median 13 months) after completing their final MRI scan.

\subsection{MR technique and protocol of dynamic enhancement}

The dynamic enhancement of MRI for bilateral breasts was performed in a $1.5 \mathrm{~T}$ superconductive MR scanner (Sonata, Siemens, Erlangen, Germany) with dedicated breast coils. Patients were prone in position with anterior chest wall abutting on the coil. To avoid motion artefact, regular and shallow breathing were instructed and mild compression of the breasts was applied. All patients received intravenous bolus injection of the contrast medium (Gadonilium-diethyltriaminepentaacetic acid, Gd-DTPA, with the dose of $0.2 \mathrm{mmole} \mathrm{kg}^{-1}$ ) at the rate of $4 \mathrm{ml}$ per second via automatic injector (Optistar MR injector, Mallinckrodt, MO, USA). A rapid scanning with 3D fast low angle shot (FLASH) pulse sequence coupled with elliptical scanning technique and partial Fourier acquisition were performed. Scan parameters were $\mathrm{TE} / \mathrm{TR}=3.1 / 12 \mathrm{~ms}$, flip angle $=25^{\circ}$, field of view $=110 \mathrm{~mm} \times 320$ to $360 \mathrm{~mm}$, image matrix $=176 \times 512$, slice thickness $=4-5 \mathrm{~mm}$, no gap, 30 slices, $14 \mathrm{~s}$ per acquisition, and total $46-50$ acquisitions. The parameter setting resulted in a pixel resolution of approximately 625-700 $\mu \mathrm{m}$. Both breasts were covered in the dynamic study for the possibility of detecting multifocal or bilateral breast cancers in our patients. Bolus injection was started at the fourth acquisition in order to achieve a steady state of magnetization and to avoid flow-related enhancement. Patients who could not tolerate scanning for approximately $45-50 \mathrm{~min}$ of the whole examination or presented with severe motion artefact in the images were excluded from our study.

\subsection{Analysis of dynamic MR data and evaluation of tumour response}

Time-intensity curves obtained from DCE MRI were analysed on a pixel-by-pixel basis with a two-compartmental model proposed by Brix et al (1991). Two angiogenic parameters, 
namely, amplitude and redistribution rate constant $\left(k_{\text {out }}\right)$, were determined by fitting the timeintensity curves (Buckley et al 1994). Amplitude represents the initial slope of enhancement and redistribution rate constant $\left(k_{\text {out }}\right)$ represents the exchange rate of the contrast agent between the intravascular and extravascular extracellular space (Buckley et al 1994, Knopp et al 1999). Colour maps of amplitude and $k_{\text {out }}$ were generated for all imaging slices.

We defined the tumour margin by manually tracing the subtraction images of dynamic enhancement showing the best margin of the tumour. Total tumour volume was determined by summing the traced tumour volumes over all slices. Tumour response was defined as the ratio of the tumour volume at the final MRI scan to the tumour volume at the first scan. For comparison, bidimensional measurement according to the World Health Organization (WHO) criteria of tumour response was also performed in each mass at the largest diameter in the axial subtraction dynamic contrast enhancement images. The tumour response assessed by tumour volume was correlated with that assessed by bidimensional measurement. The responders were defined as the patients who showed greater than $50 \%$ reduction in tumour volume whereas non-responders were those showing less than $50 \%$ reduction. The definition of region of interest (ROI) or tumour margin and the largest tumour dimension were performed by one author (Chang) who had 4 years of experience in breast MRI. Data analysis was performed with Matlab 6.1 (MathWorks Inc., Natick, MA, USA) and Mathematica 4.1 (Wolfram Media, Inc., Champaign, IL, USA).

Histograms of DCE MRI-derived angiogenic parameters of the whole tumour, including amplitude, $k_{\text {out }}$ and peak enhancement were also obtained. To describe distribution patterns of tumour compositions, we calculated statistical indices including mean, standard deviation, skewness (degree of asymmetry of a distribution) and kurtosis (degree of peakedness of a distribution) from the histograms of each angiogenic parameter. Images of different patients were considered to be independent measurements.

To determine the predictive value of the statistical indices, we analysed the association between tumour response and initial values of statistical indices. We also analysed the association between tumour response and the ratio of statistical indices after the first cycle of chemotherapy to the same indices before chemotherapy. To characterize the change of statistical indices over the whole course of chemotherapy, we analysed the association between tumour response and the ratio of statistical indices after completing the whole course of chemotherapy to the same indices before chemotherapy. The test of correlation between statistical indices and tumour response was performed with the linear regression model, and the $p$ value of less than 0.05 was considered significant.

\section{Results}

There were $61 \mathrm{MR}$ studies in total with a minimum of three studies for each case. The interval between the first and final MR studies was $4.88 \pm 2.87$ months (median 4.2 months). Excellent correlation was found between tumour response assessed by tumour volume and that by bidimensional measurement $(r=0.93)$. The numbers of responders and non-responders were, respectively, 8 and 5 by volume measurement, and 7 and 6 by WHO criteria. The tumour size was $188.88 \pm 140.54 \mathrm{ml}$ before chemotherapy and was $70.27 \pm 73.90 \mathrm{ml}$ after completing the course of chemotherapy.

Colour maps of amplitude showed that better tumour response was marked by a change from heterogeneous to homogeneous tumour compositions and a decrease in peak enhancement (figure 1(a)). In contrast, there was little perceptible change of internal tumour compositions in the non-responders (figure 2(b)). In the responders the histograms of amplitude showed a progressive decrease and left shift of the peak, and narrowing of the base (figure 1(b)). 

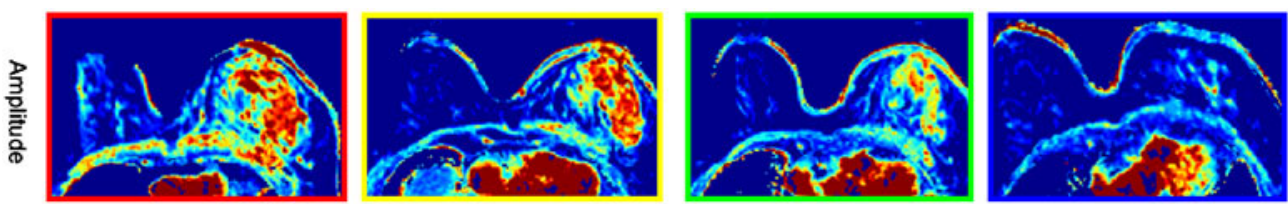

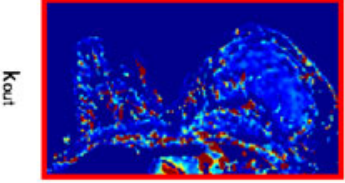

Before chemotherapy

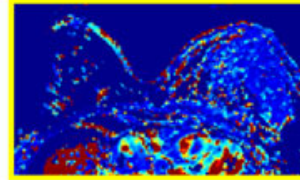

After first chemotherapy

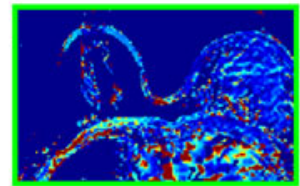

After 2nd chemotherapy

(a)

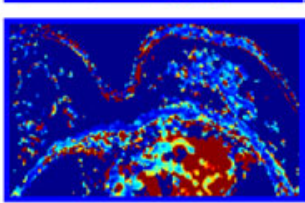

After final chemotherapy

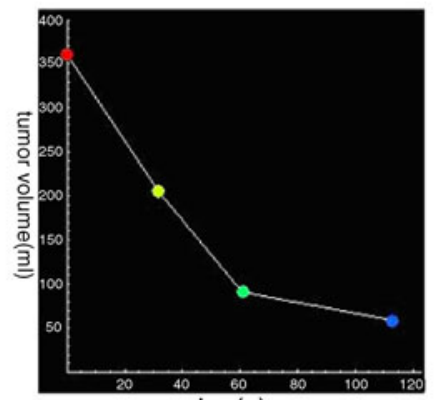

$\operatorname{day}(\mathrm{s})$

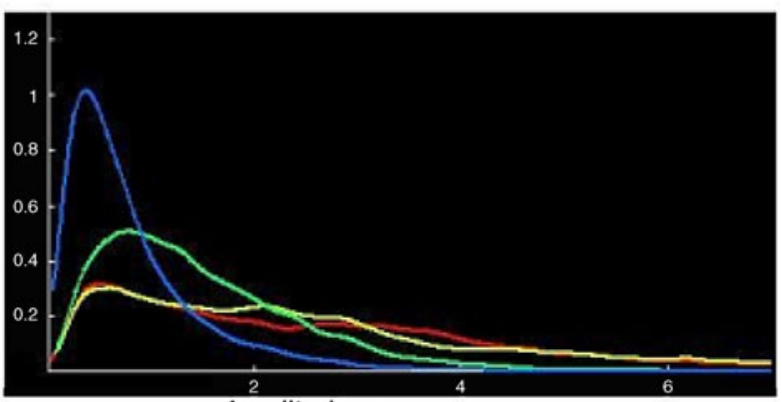

(b)

Figure 1. An example of a responder. (a) A large left breast cancer containing heterogeneous compositions before chemotherapy (as shown in the amplitude map in the upper row, the first on the left) becomes more homogeneous following serial courses of chemotherapy (upper row, from left to right). In contrast, there is no significant change of the tumour compositions based on $k_{\text {out }}$ map during serial courses of chemotherapy (lower row, from left to right). The final tumour response is $16 \%$ in this case. (b) Serial tumour volume change (left panel) and normalized histograms of amplitude of the whole tumour (right panel). There is a marked decrease of tumour size (left panel, red dot followed by yellow, green and blue dot). Correspondingly, the initial histogram of amplitude appears flat and wide (right panel, red curve). Serial follow-up during the course of chemotherapy reveals gradual left shift of the peak of the histograms and more uniform distribution in the final MR study (right panel, blue curve).

Such change was not observed in the histograms of $k_{\text {out }}$ or histograms of both amplitude (figure $2(\mathrm{~b})$ ) and $k_{\text {out }}$ in the non-responders.

As listed in table 1, kurtosis and standard deviation of amplitude before chemotherapy correlated with tumour response ( $r=0.63$ and $r=0.61$, respectively). After the first course of chemotherapy the changes in standard deviation of amplitude, and in kurtosis and standard deviation of $k_{\text {out }}$ correlated with tumour response ( $r=0.79,0.57$ and 0.57 , respectively). In comparing the final preoperative MRI study with the initial study, better tumour response was indicated by a significant change in amplitude, showing an increase in kurtosis $(r=0.62)$, and a decrease in mean $(r=0.84)$ and standard deviation $(r=0.77)$, and in peak enhancement showing a decrease in mean $(r=0.71)$.

\section{Discussion}

In this study, we analysed signal time curves on a pixel-by-pixel basis over bilateral whole breasts. This approach allowed complete mapping of tumour angiogenic compositions and 


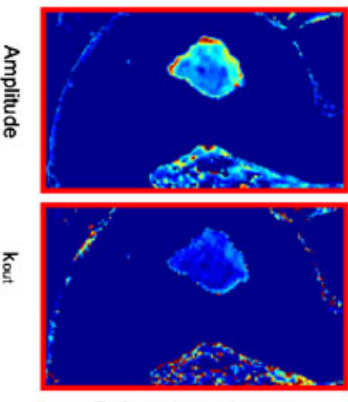

Before chemotherapy

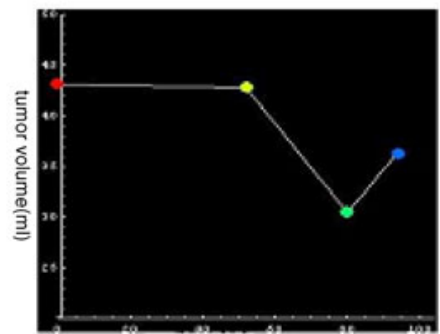

$\operatorname{day}(\mathrm{s})$
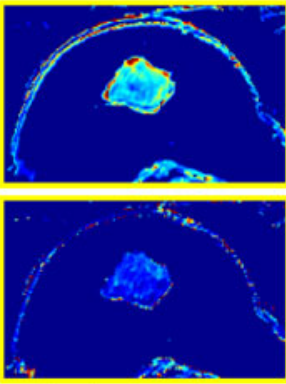

After first chemotherapy

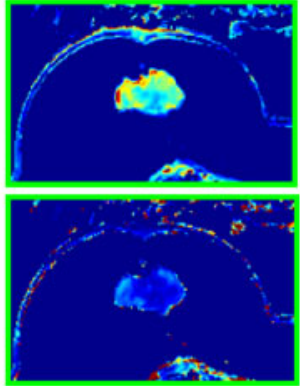

After 2nd chemotherapy

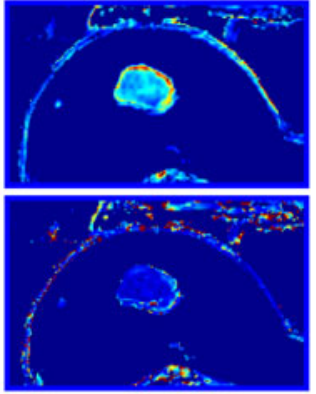

After 3nd chemotherapy

(a)

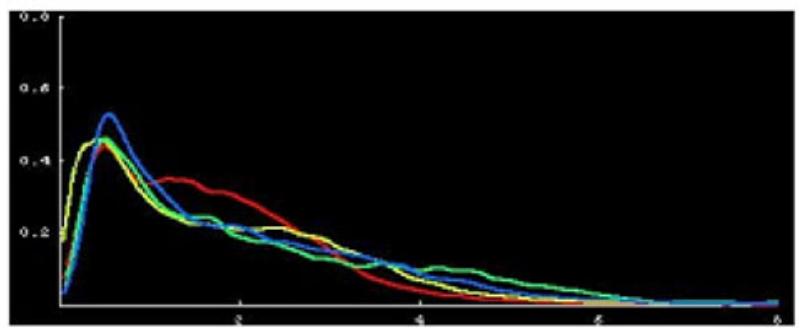

(b)

Amplitude

Figure 2. An example of a non-responder. (a) Serial colour mapping of both amplitude (upper row) and $k_{\text {out }}$ (lower row) shows rather homogeneous compositions before chemotherapy (the first on the left, upper and lower rows). No significant perceptible change of internal tumour compositions is found during serial courses of chemotherapy on amplitude maps (upper row, from left to right) and $k_{\text {out }}$ maps (lower row, from left to right). The final tumour response is $84 \%$ in this case (b) Serial volume change of tumour (left panel) and normalized histograms of amplitude of the whole tumour (right panel). The histograms of amplitude during the course of chemotherapy show little change in distribution over the whole course of chemotherapy.

Table 1. Correlation between different MRI-derived angiogenic parameters and tumour response at different time points.

\begin{tabular}{|c|c|c|c|c|c|c|c|c|c|c|c|c|}
\hline & \multicolumn{4}{|c|}{ Before $\mathrm{C} / \mathrm{T}$} & \multicolumn{4}{|c|}{ After first $\mathrm{C} / \mathrm{T}$} & \multicolumn{4}{|c|}{ After final $\mathrm{C} / \mathrm{T}$} \\
\hline & Mean & SD & Kurtosis & Skewness & Mean & SD & Kurtosis & Skewness & Mean & SD & Kurtosis & Skewness \\
\hline \multicolumn{13}{|c|}{ Amplitude versus tumour response } \\
\hline$r$ & 0.42 & $0.61^{*}$ & $0.63^{*}$ & 0.45 & 0.24 & $0.79^{*}$ & 0.41 & 0.46 & $0.84^{*}$ & $0.77^{*}$ & $0.62^{*}$ & 0.49 \\
\hline$p$ & 0.14 & $0.03^{*}$ & $0.02^{*}$ & 0.12 & 0.43 & $0.001^{*}$ & 0.16 & 0.11 & $0.0003^{*}$ & $0.002 *$ & $0.02^{*}$ & 0.09 \\
\hline \multicolumn{13}{|c|}{$k_{\text {out }}$ versus tumour response } \\
\hline$r$ & 0.1 & 0.1 & 0.41 & 0.46 & 0.26 & $0.57^{*}$ & $0.57^{*}$ & 0.33 & 0.24 & 0.41 & 0.5 & 0.35 \\
\hline$p$ & 0.70 & 0.76 & 0.16 & 0.11 & 0.38 & $0.04^{*}$ & $0.04^{*}$ & 0.26 & 0.43 & 0.16 & 0.08 & 0.25 \\
\hline \multicolumn{13}{|c|}{ Peak enhancement versus tumour response } \\
\hline$r$ & 0.32 & 0.00 & 0.37 & 0.26 & 0.1 & 0.22 & 0.00 & 0.24 & $0.71^{*}$ & 0.37 & 0.54 & 0.2 \\
\hline$p$ & 0.28 & 0.93 & 0.22 & 0.38 & 0.74 & 0.44 & 0.84 & 0.40 & $0.01^{*}$ & 0.21 & 0.06 & 0.51 \\
\hline
\end{tabular}

SD: standard deviation, C/T: chemotherapy, $r$ : correlation coefficient; ${ }^{*} p<0.05$.

facilitated visual perception of tumour response to chemotherapy. We further characterized tumour heterogeneity in terms of histograms of angiogenic parameters. Using this metric we 
found that tumour heterogeneity in LABC was associated with tumour response to neoadjuvant chemotherapy.

Neoadjuvant chemotherapy of LABC can result in satisfactory local control, and even increased overall survival, especially in patients with a complete clinical or histopathological response (Eltahir et al 1998). Mammography has been widely accepted as an important tool to detect breast cancer but plays a limited role in assessing the response of breast cancer to neoadjuvant chemotherapy. Its efficacy is even inferior to physical palpation in patients with dense breasts (Vinnicombe et al 1996, Weatherall et al 2001). Although serial biopsy during neoadjuvant chemotherapy provides a window to observe tumour response over time, a small amount of tissue specimen is subjected to sampling bias in the context of heterogeneous internal compositions. DCE MRI has been extensively studied in patients with breast tumours, and is found valuable in monitoring tumour residura, finding unsuspected or multifocal tumours, differentiating benign from malignant lesions, and characterizing tumour angiogenesis (Gilles et al 1994, Mussurakis et al 1997, Tofts et al 1995, Knopp et al 1999, Taylor et al 1999, Fischer et al 1999, Kuhl et al 1999, Carriero et al 2002, Hulka et al 1997). Recent studies also showed that DCE MRI is potentially useful in assessing tumour response to neoadjuvant chemotherapy (Abraham et al 1996, Rieber et al 1997, 2002, Wasser et al 2003, Delille et al 2003). It has been demonstrated that the kinetics of Gd-DTPA uptake is contributed from microvessel density (MVD), vessel size and permeability (Buadu et al 1996, Carriero et al 2002, Hulka et al 1997). To better explain the kinetics of Gd-DTPA uptake, a pharmacokinetic two-compartment model was proposed to characterize time-intensity curves in terms of amplitude, redistribution rate constant $\left(k_{\text {out }}\right)$ (Knopp et al 1999, Brix et al 1991, Buckley et al 1994). In this model, the amplitude was related to MVD, and exchange rate $k_{\text {out }}$ was related to vascular permeability that was proportional to vascular endothelial growth factor (VEGF) (Knopp et al 1999). Using these MRI-dervied angiogenic parameters Knopp et al (1999) were able to subclassify malignant breast tumours. The pixel-by-pixel analysis of Gd-DTPA uptake time curves not only improves the specificity of diagnosis, it can produce parametric images that provide information about tumour heterogeneity, permeability and vascularity (Mussurakis et al 1997). Therefore, DCE MRI is a useful tool to monitor the response of tumour angiogenesis and tailor the choice of therapeutic regimens for patients with LABC.

Most of the DCE MRI studies analysed averaged signal time curves from a tumour region in a single slice. However, this approach cannot indicate the heterogeneity of tumour compositions that may be a key factor affecting tumour response to chemotherapy. In our study, we compared the tumour response with the dynamics of angiogenic compositions during serial neoadjuvant chemotherapy. Better tumour response was associated with a larger change of tumour compositions from heterogeneous to homogeneous distributions, and larger decrement of peak enhancement over the whole tumour. Before chemotherapy, lower kurtosis and higher standard deviation of amplitude correlated with better tumour response $(r=0.63$ and $r=$ 0.61 , respectively). After the first course of chemotherapy, better responders showed larger decrease of standard deviation of amplitude $(r=0.79)$, larger increase of kurtosis and decrease of standard deviation of $k_{\text {out }}$ ( $r=0.57$ and 0.57 , respectively). In the final MR study, better responders showed larger increase of kurtosis $(r=0.62)$, decrease of mean $(r=0.84)$ and standard deviation $(r=0.77)$ of amplitude and decrease of mean of peak enhancement $(r=$ 0.71). These results suggest that the change in internal compositions may arise from different susceptibility of individual compositions to chemotherapy. Particularly, compositions with higher amplitude may be more susceptible to chemotherapy.

Ellis et al (1997) found that during neoadjuvant treatment a measurable increase in apoptosis occurred in breast tumours within $24 \mathrm{~h}$ after the start of chemotherapy; this increase 
was associated with concomitant decrease in proliferation of tumour cells (Cleator et al 2002). Gilles et al (1994) showed that tumour response to primary chemotherapy was related to the replacement of tumour cells with loose fibrosis and that the proportion of tumour cells and fibrotic change correlated well with the pattern of enhancement of residual tumour. These studies also indicate that there are substantial alterations of internal compositions in the breast cancer undertaking chemotherapy. Relationships between histological changes and MRIdervied tumour angiogenic parameters are currently under active investigation in our lab.

A recent study by Wasser et al (2003) revealed the potential capability of DCE MRI to assess therapeutic effects of neoadjuvant chemotherapy in breast cancer. On the basis of the two-compartment model, they showed good correlation between tumour diameter measured by histopathology and MRI $(r=0.7, p<0.003)$. Furthermore, a reduction of tumour size after chemotherapy was associated with a decrease of both $k_{\text {ep }}$ (equivalent to $k_{\text {out }}$ ) (Padhani and Husband 2001) and amplitude, where $k_{\mathrm{ep}}$ was a more sensitive indicator that began to drop right after the first cycle of chemotherapy. On the other hand, the reduction of contrast enhancement was not a sign of tumour responsiveness (Wasser et al 2003). In our study, tumours with better response were more heterogeneous in the histograms of amplitude before chemotherapy (table 1). Histograms of $k_{\text {out }}$ showed increased kurtosis and decreased standard deviation after the first course of chemotherapy. These findings suggest that compositions of tumour angiogenesis might become more homogeneous under effective chemotherapy. However, we did not find significant decrease in the mean values of $k_{\text {out }}$ or amplitude. The disparity might arise from variable chemotherapy regimens employed in our study. Further investigation with randomized controlled study will be needed to draw a more conclusive result. Comparing with amplitude, we found that the response of $k_{\text {out }}$ was rather inconsistent, suggesting that $k_{\text {out }}$ might respond differently to different chemotherapy regimens. Delille et al (2003) reported that DCE breast MRI using extraction flow product (EFP) could provide important functional information regarding changes in tumour angiogenesis due to neoadjuvant chemotherapy. Similar to the behaviour of $k_{\text {out }}$, changes of EFP appeared to be dependent on the chemotherapeutic regimens.

Motion artefact with consequent pixel shift may cause error in data fitting of signal intensity-time curve. Minor motion artefact during dynamic study with free breathing is inevitable (Gilles et al 1994, Tofts et al 1995, Knopp et al 1999, Taylor et al 1999, Fischer et al 1999, Kuhl et al 1999). Manual tumour definition may also cause interobserver and intraobserver variation. These errors, however, may not cause significant change in statistical indices because LABC allows large numbers of pixels to be sampled for analysis. Although our results revealed the possibility of using internal MR parametric composition to predict tumour response to neoadjuvant chemotherapy, large number series with histopathologic correlation is warranted for further validation.

In conclusion, we demonstrated the potential usefulness of parametric histograms derived from DCE MRI to characterize the heterogeneity of tumour compositions and to study the response of tumour angiogenesis to neoadjuvant chemotherapy. Using this method, in vivo angiogenic compositions of the whole breast tumour mass can be monitored by serial MRI studies during the whole course of neoadjuvant chemotherapy. This method might be clinically useful in understanding pathophysiologic changes, predicting tumour response and guiding therapeutic approach.

\section{Acknowledgments}

The study was supported in part by the National Science Council (NSC 90-2314-B-002-320) and the National Taiwan University Hospital (91-S064). 


\section{References}

Abraham D C, Jones R C, Jones S E, Cheek J H, Peter G N, Knox S M, Grant M D, Hampe D W, Savino D A and Harms S E 1996 Evaluation of neoadjuvant chemotherapeutic response of locally advanced breast cancer by magnetic resonance imaging Cancer 78 91-100

Boetes C, Mus R, Holland R, Barentsz J O, Strijk S P, Wobbes T, Hendriks J H and Ruys S H 1995 Breast tumors: comparative accuracy of MR imaging relative to mammography and US for demonstrating extent Radiology $197743-7$

Brix G, Semmler W, Port R, Schad L R, Layer G and Lorenz W J 1991 Pharmacokinetic parameters in CNS Gd-DTPA enhanced MR imaging J. Comput. Assist. Tomogr. 15 621-8

Buadu L D, Murakami J, Murayama S, Hashiguchi N, Sakai S, Masuda M, Toyoshima S, Kuroki S and Ohno S 1996 Breast lesions: correlation of contrast medium enhancement patterns on MR images with histopathologic findings and tumor angiogenesis Radiology 200 639-47

Buckley D L, Kerslake R W, Blackband S J and Horsman A 1994 Quantitative analysis of multi-slice Gd-DTPA enhanced dynamic MR imaging using an automated simplex minimization procedure Magn. Reson. Med. 32 $646-51$

Carriero A, Ambrossini R, Mattei P A, Angelucci D and Bonoma L 2002 Magnetic resonance of the breast: correlation between enhancement patterns and microvessel density in malignant tumors J. Exp. Clin. Cancer Res. 21 $83-7$

Chie W C, Chang S H, Huang C S, Tzeng S J, Chen J H, Fan B Y and Chang K J 2002 Prognostic factors for the survival of Taiwanese breast cancer patients J. Formos. Med. Assoc. 101 98-103

Cleator S, Parton M and Dowsett M 2002 The biology of neoadjuvant chemotherapy for breast cancer Endocr. Relat. Cancer 9 183-95

Delille J P, Slanetz P J, Yeh E D, Halpern E F, Kopans D B and Garrido L 2003 Invasive ductal breast carcinoma response to neoadjuvant chemotherapy: noninvasive monitoring with functional MR imaging-pilot study Radiology 228 63-9

Ellis P A, Smith I E, McCarthy K, Detre S, Salter J and Dowsett M 1997 Preoperative chemotherapy induces apoptosis in early breast cancer Lancet 349849

Eltahir A, Heys S D, Hutcheon A W, Starkar T K, Smith I, Walker L G, Ah-See A K and Eremin O 1998 Treatment of large and locally advanced breast cancer using neoadjuvant chemotherapy Am. J. Surg. 175 127-32

Fischer U, Kopla L and Grabbe E 1999 Breast carcinoma: effect of preoperative contrast-enhanced MR imaging on the therapeutic approach Radiology 213 881-8

Gilles R, Guinebretière J M, Toussaint C, Spielman M, Rietjens M, Petit J Y, Contesso G, Masselot J and Vanel D 1994 Locally advanced breast cancer: contrast-enhanced subtraction MR imaging of response to preoperative chemotherapy Radiology $191633-8$

Heys S D, Eremin J M, Sarkar T K, Hutcheon A W, Ah-See A and Eremin O 1994 Role of multimodality therapy in the management of locally advanced carcinoma of the breast J. Am. Coll. Surg. 179 493-504

Hulka C A, Edmister W B, Smith B L, Tan L, Sgroi D C, Campbell T, Kopans D B and Weisskoff R M 1997 Dynamic echo-planar imaging of the breast: experience in diagnosing breast carcinoma and correlation with tumor angiogenesis Radiology 205 837-42

Knopp M V et al 1999 Pathophysiologic basis of contrast enhancement in breast tumors J. Magn. Reson. Imaging 10 260-6

Koning C and Hart G 1998 Long-term follow-up of a randomized trial on adjuvant chemotherapy and hormonal therapy in locally advanced breast cancer Int. J. Radiat. Oncol. Biol. Phys. 41 397-400

Kuhl C K, Mielcareck P, Klaschik S, Leutner C, Wardelmann E, Gieseke J and Schild H H 1999 Dynamic breast MR imaging: are signal intensity time course data useful for differential diagnosis of enhancing lesions? Radiology 211 101-10

Mumtaz H, Hall-Craggs M, Davidson T, Walmsley K, Thurell W, Kissin M W and Taylor I 1997 Staging of symptomatic primary breast cancer with MR imaging Am. J. Roentgenol. 169 417-24

Mussurakis S, Buckley D L, Drew P J, Fox J N, Carleton P J, Turnbull L W and Horsman A 1997 Dynamic MR imaging of the breast combined with analysis of contrast agent kinetics in the differentiation of primary breast tumors Clin. Radiol. 52 516-26

Padhani A R and Husband J E 2001 Dynamic contrast-enhanced MRI studies in oncology with an emphasis on quantification, validation and human studies Clin. Radiol. 56 607-20

Pierce L, Adler D, Helvie M, Lichter A and Merajver S 1996 The use of mammography in breast preservation in locally advanced breast cancer Int. J. Radiat. Oncol. Biol. Phys. 34 571-7

Rieber A, Brambs H J, Gabelmann A, Heilmann V, Kreienberg R and Kühn T 2002 Breast MRI for monitoring response of primary breast cancer to neo-adjuvant chemotherapy Eur. Radiol. 12 1711-9 
Rieber A, Zeitler H, Rosenthal H, Görich J, Kreienberg R, Brambs H J and Tomczak R 1997 MRI of breast cancer: influence of chemotherapy on sensitivity Br. J. Radiol. $70452-8$

Rodenko G N, Harms S E, Pruneda J M, Farrell R S Jr, Evans W P, Copit D S, Krakos P A and Flamig D P 1996 MR imaging in the management before surgery of lobular carcinoma of the breast: correlation with pathology Am. J. Roentgenol. 167 1415-9

Tabar L, Yen M F, Vitak B, Chen H H, Smith R A and Duffy S W 2003 Mammography service screening and mortality in breast cancer patients: 20-year follow-up before and after introduction of screening Lancet 361 1405-10

Taylor J S, Tofts P S, Phil D, Port R, Evelhoch J L, Knopp M, Reddick W E, Runge V M and Mayr N 1999 MR imaging of tumor microcirculation: promise for the new millennium J. Magn. Reson. Imaging 10 903-7

Tofts P S, Berkowitz B and Schnall M 1995 Quantitative analysis of dynamic Gd-DTPA enhancement in breast tumors using a permeability model Magn. Reson. Med. 33 564-8

Vinnicombe S, MarVocar A D, Guy R L, Sloane J P, Powles T J, Knee G and Husband J E 1996 Primary breast cancer: mammographic changes after neoadjuvant chemotherapy with pathologic correlation Radiology $198333-40$

Wasser K, Klein S K, Fink C, Junkermann H, Sinn H P, Zuna I, Knopp M V and Delorme S 2003 Evaluation of neoadjuvant chemotherapeutic response of breast cancer using dynamic MR with high spatial temporal resolution Eur. Radiol. 13 80-7

Weatherall P T, Evans G F, Metzger G J, Saborrian M H and Leitch A M 2001 MRI vs. histologic measurement of breast cancer following chemotherapy: comparison with X-ray mammography and palpation J. Magn. Reson. Imaging 13 868-75

Weidner N, Semple J P, Welch W R and Folkman J 1991 Tumor angiogenesis and metastasis—correlation in invasive breast cancer N. Engl.J. Med. 324 1-8 